\title{
An Open Model for Generating High Resolution Wind Power Production Scenarios
}

\author{
$1^{\text {st }}$ Elis Nycander \\ Division of Electric Power and Energy Systems \\ KTH Royal Institute of Technology \\ Stockholm, Sweden \\ elisn@kth.se
}

\author{
$2^{\text {nd }}$ Lennart Söder \\ Division of Electric Power and Energy Systems \\ KTH Royal Institute of Technology \\ Stockholm, Sweden \\ lsod@kth.se
}

\begin{abstract}
Efficient integration of variable renewable energy (VRE) such as wind power into power systems requires methods for power system operation planning that account for VRE uncertainty and variability. This has motivated extensive research into unit commitment (UC) and optimal power flow (OPF) formulations with VRE uncertainty. However, these formulations are often tested using significantly simplified representations of VRE production. We seek to address this issue by providing a model for generating realistic wind power scenarios using real production and forecast data. The scenarios are generated using 5-min production and 30-min forecast data for real wind farms from Australia. The model captures the empirical distribution of the forecast errors and the covariance between different wind farms. The high time resolution of the production data also allows the recreation of the high-frequency (5-min) component of wind power production. The resulting model is openly available, and can be used to generate wind power scenarios for use in formulations for operation planning of power systems (UC/OPF) considering wind uncertainty and intra-hour variability. The scenarios can be tailored according to preferences for, e.g., the number of wind farms and their geographical dispersion.

Index Terms-Wind power scenarios, 5-min resolution, stochastic optimization, quantile regression
\end{abstract}

\section{INTRODUCTION}

Efficiently integrating large amounts of variable renewable energy (VRE), such as wind power, into power systems will require methods for power system operation that account for the uncertainty and variability inherent in VRE production. This has motivated a lot of research into unit commitment (UC) and optimal power flow (OPF) formulations with VRE uncertainty [1]-[3]. Most of these formulations consider wind power as the main source of uncertainty.

The wind uncertainty can be modelled in different ways. Broadly defined three types of formulations can be identified [2]: 1) stochastic formulations which represent the wind uncertainty using a set of scenarios and optimize the expected value of costs over the scenarios, 2) formulations with chanceconstraints, e.g., security constraints which are fulfilled with a certain probability, and 3) robust formulations which ensure that the unit schedules can accommodate any outcome of wind production within a given uncertainty range.

Stochastic formulations [4]-[7] are dependent on the ability to generate realistic and representative wind power scenarios [4]. However, the methods used to generate the scenarios in the literature vary. Sahin et al. [6] start from the expected wind production and model the forecast error as a normal distribution with increasing variance over the planning horizon, while Uçkun et al. [7] use a method based on probabilistic forecasting. On the other hand, Papavasiliou et al. [4] model wind speeds using an auto-regressive model and then convert these to wind power production using power curves based on aggregated historical data. Wu et al. [5] also use power curves to convert wind speeds to wind power production, but obtain the wind speeds directly from a Weibull distribution.

Chance-constrained formulations define constraints which may be violated with a specified probability, based on underlying assumptions about the uncertainty distributions. Commonly multivarate normal distributions are assumed [8]-[10], which allows for tractable reformulations of the chance constraints. The chance constraints can also be approximated using scenarios as in [8].

Finally, robust optimization formulations use an uncertainty set within which the wind production can take its worst possible value [11]-[16]. This uncertainty range can be determined based on historical production [11], [12] and/or set to a fixed fraction of the expected wind production [13], [14]. Thus no information about the underlying probability distribution is required in the formulation itself.

Even though the representation of the uncertainty is different between different formulations types, realistic wind power scenarios are needed for evaluating the performance of the commitment and dispatch decisions obtained from the formulation. This is done by out-of-sample simulation, i.e., simulating the system for a large number of realizations of the uncertain wind production, and evaluating the resulting costs, including costs of emergency actions such as re-dispatch and load shedding needed to cope with the uncertainty realization. Thus, although chance-constrained and robust formulations do not require scenarios in the formulation itself, they should still be evaluated using realistic wind power scenarios. However, the assumptions made when doing out-of-sample simulation vary widely. Commonly the forecast errors are assumed to follow a normal distribution [13], [14], although other distributions such as the uniform [13] or beta distribution [15] have also been used. In [10] the Laplace, logistic, and Weibull distributions are tested for out-of-sample simulation, while 
Morales-España et al. use a multivariate normal distribution to account for inter-temporal correlations and correlations between wind farms [16].

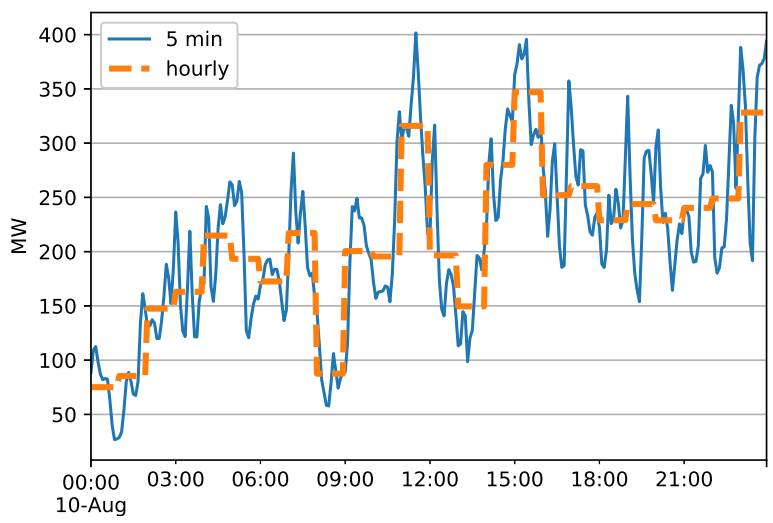

Figure 1. Production of the Macarthur Wind Farm in Victoria, Australia during August 10, 2019. Notice the large fluctuations of the 5-min production data compared to the hourly averages.

The large variety of methods used to model wind power uncertainty makes comparison of different formulations difficult. Additionally, the formulations in [4]-[16] all use hourly wind power scenarios. Since the intra-hour variability of wind power can be high (Fig. 1), this means that an important aspect of system robustness is not captured by hourly simulations. For this reason, Morales-Espana et al. [17] use a 5-min simulation for evaluating the performance of the unit schedules obtained by their formulation. However, the scenarios used do not have significant intra-hour variability.

In this paper, we describe a method for obtaining realistic 5-min wind power scenarios, and provide an open model that can be used to easily generate an arbitrary number of scenarios from the historical forecast for a given day. The model uses 5min production data and 30-min forecast data from Australian wind farms, made available by AEMO [18], and uses a method similar to the one presented by Pinson et al. [19] to estimate the distribution of forecast errors, considering the empirical distribution of forecast errors and covariance between different wind farms. The high resolution of the production data allows estimation of the high-frequency component of wind power production, which is recreated using filtered white noise and added to the scenarios. This allows the creation of realistic scenarios with 5-min time resolution, thus also capturing the intra-hour wind power variations. The model is open and available on Github ${ }^{1}$. Since it is based on open data from real wind farms it is possible modify the model to include the desired number of wind farms or change their geographical dispersion. By providing this model we hope to facilitate evaluation of UC/OPF models with wind uncertainty, thereby helping their development.

The remainder of the paper is organized as follows: Section II describes the data used, Section III describes the fitting of the forecast error distributions and Section IV describes the

\footnotetext{
${ }^{1}$ https://github.com/ElisNycander/wind_scenarios
}

fitting of the high frequency component of wind production. Finally, Section V performs a validation and describes the application of the scenarios, and Section VI concludes.

\section{DESCRIPTION OF DATA}

The Australian Electricity Market Operator (AEMO) makes dispatch data available on its NEMWEB portal [18]. 5-min production data for all units, including wind farms, are available under "Dispatch - SCADA" and 30-min forecasts for wind farms are available under "PreDispatch Summary - Public". Forecasts are published for a 24 hour period, starting at 4:30 am on the delivery day $\mathrm{D}$ until 4:00 am on day $\mathrm{D}+1$. The first forecast for the 24 hour period is released at 12:30 pm on D-1 and then the forecasts are updated every 30 minutes, the last forecast being published at 3:30 am on $\mathrm{D}+1$. Thus forecasts for the full 24 hour period are available with a maximum lead time of 16 hours and a minimum lead time of 30 minutes.

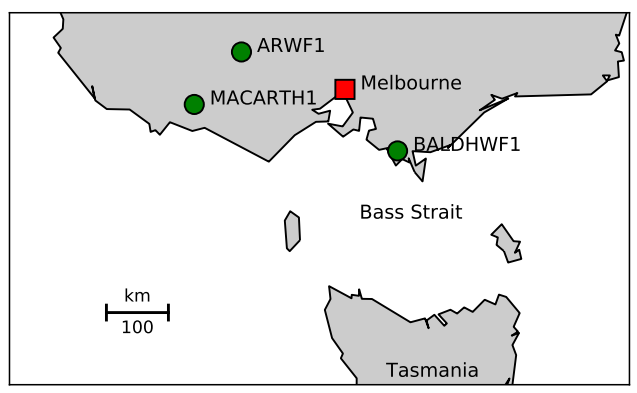

Figure 2. Wind farms included in model.

In this paper data from 2019-08-01 until 2020-02-28 has been used for fitting the model. All results shown are for forecasts with a lead time of $30 \mathrm{~min}$, which means that the whole 24-hour forecast was made 30-minutes before the first time step of the 24-hour period. The wind farms chosen for the model were the Ararat Wind Farm (ARWF1, $240 \mathrm{MW}$ ), the Macarthur Wind Farm (MACARTH1, $420 \mathrm{MW}$ ), and the Bald Hills Wind Farm (BALHWF1, 107 MW), all located in Victoria, Australia (Fig. 2). However, the wind farms can be changed and the model re-fitted.

The AEMO data is available as csv files, but has been stored in an Sqlite database to allow quick access from Python. A subset of the data used, from 2019-08-01 until 2019-10-31, is available as an Sqlite database together with the Github repository ${ }^{1}$, which may be used by users who wish to generate scenarios from the existing model.

\section{Fitting ForecAst ERROR Distribution}

The forecast error distribution is fit separately for each wind farm using the 30-min resolution forecast data. Notice that both the production and forecast data has been normalized with the installed capacity (computed as the maximum production value during the considered period). To remove high-frequency noise present in the 5-min production data it is first low-pass filtered using a Butterworth filter of 6th order and a cut-off frequency of 1 hour $^{-1}$, which corresponds to the Nyquist 

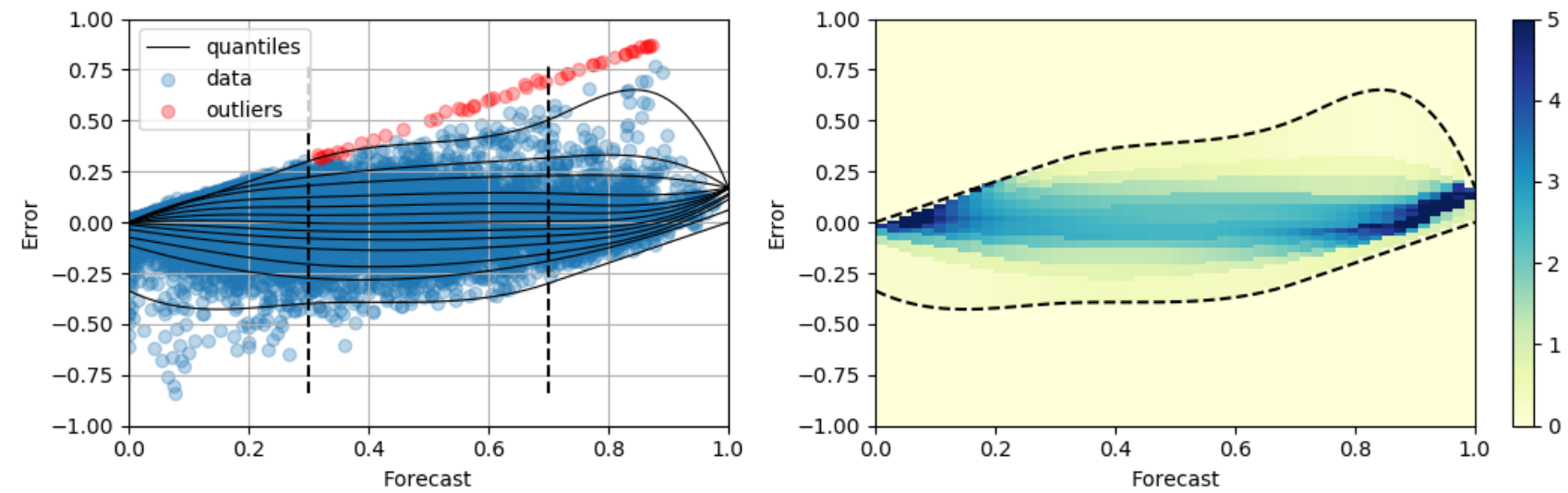

Figure 3. Distribution of the (relative) forecast error for ARWF1. Left figure shows fitted quantile curves and right figure shows the pdf of the resulting parameterized distribution.
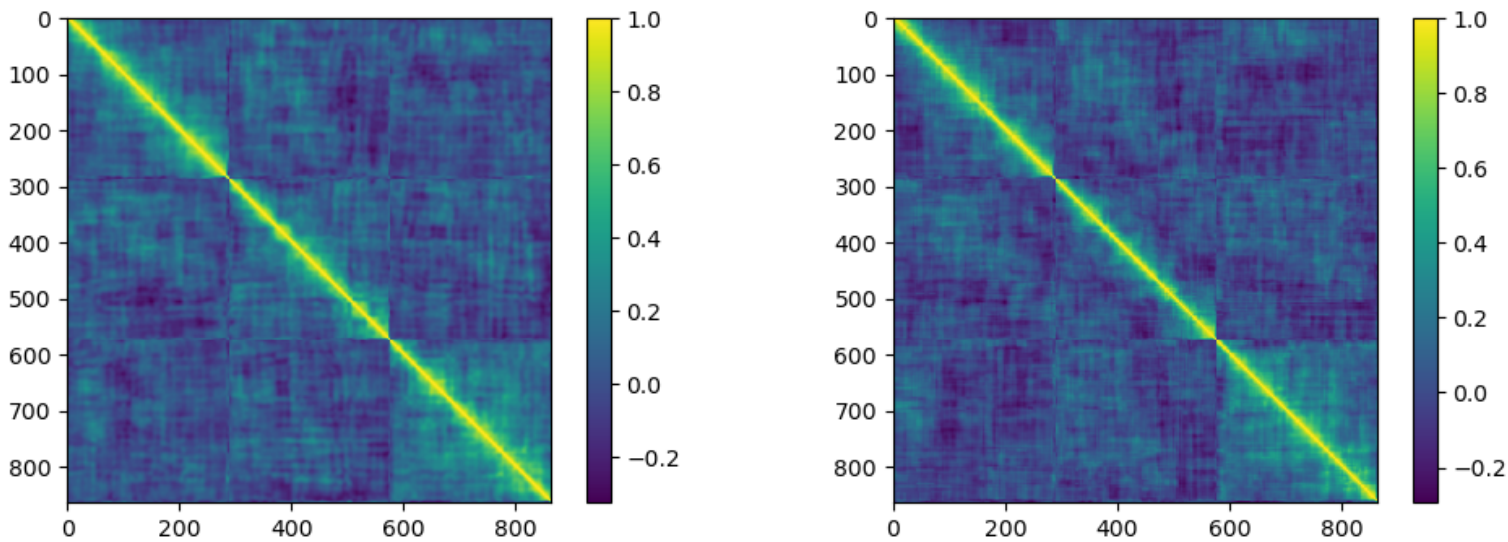

Figure 4. Coorelation matrix for original errors (left) and errors mapped to normal distribution (right). The wind farms, in order, are ARWF1, MACARTH1, and BALDHWF1. Three wind farms and $24 \cdot 12$ periods for each wind farm gives a total of $3 \cdot 288=864$ variables.

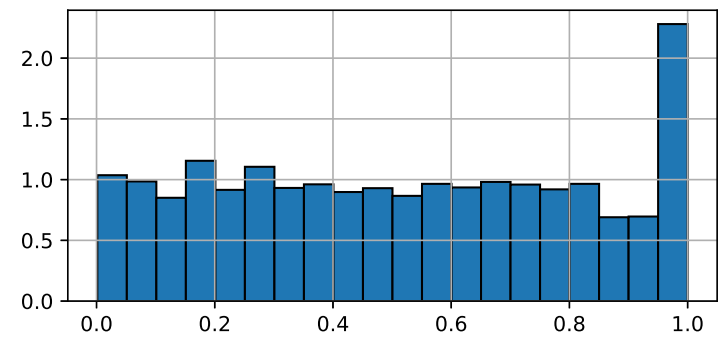

Figure 5. Distribution of values of $F_{e r}(x)$ for ARWF1

frequency of the forecast data. Fig. 3 shows the forecast error distribution for ARWF1, where an over-prediction is defined as a positive forecast error. Notice that there is a large number of points close to the identify line where error=forecast, also for higher forecast values. For forecasts larger than 0.3 such points (where the difference between the forecast and the error was less than $2 \%$ ) were excluded from the fit, so as not to create a too wide distribution of forecast errors.
To approximate the distribution, curves representing the $1 \%$, $5 \%, 10 \%, 20 \%, \ldots, 90 \%, 95 \%$, and $99 \%$ quantiles were fit using quantile regression [20], as proposed in [19]. Each curve was parameterized using three cubic splines, where the dashed lines in Fig. 3 show the spline boundaries. All spline curves for a wind farm were fit using a single (LP) optimization, which allowed additional constraints to ensure that the spline curves were monotonically increasing at the boundaries, i.e. for a forecast of 0 or 1 . Between the quantile curves the cumulative density function (cdf) of the forecast error was assumed to increase linearly, corresponding to a constant probability density function (pdf). However, notice that the fitted curves for $1 \%$ and $99 \%$ were assumed to represent the $0 \%$ and $100 \%$ quantiles, in order to remove the possibility of extreme outliers such as the the values excluded from the fit. Also, the $99 \%$ curve was fixed to the forecast error boundary given by the identity line for a forecast below 0.3 , and similarly for the $1 \%$ quantile line for a forecast above 0.7. The reason is that for a low forecast a zero production outcome should be within the probability distribution, and similarly for a high forecast a 

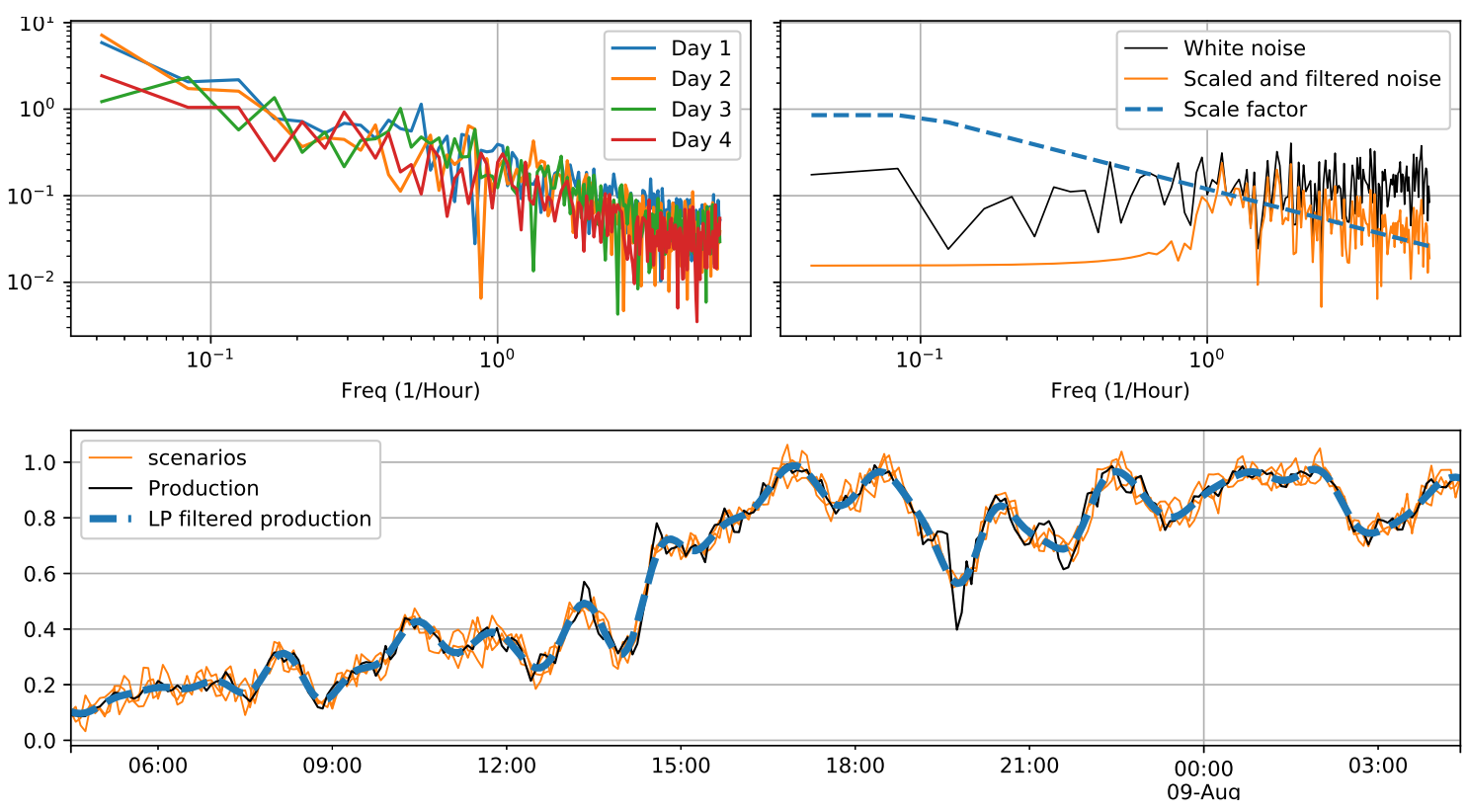

Figure 6. Fitting of high-frequency component of production for ARWF1 for bin 3. Top: spectra (ffts) for production on 4 days (left) and the fitted scaling function with the spectrum of white noise and the resulting scaled spectrum (right). Bottom: three scenarios generated by adding different realizations of the high-frequency component to the low-pass filtered production.

production outcome at maximum capacity should be possible. Together the quantile curves give a complete description of the forecast error distribution, the pdf of which is shown in the right of Fig. 3.

Given the marginal distribution of forecast errors, $F_{e r}(x)=$ $P$ (error $\leq \mathrm{x} \mid$ forecast $)$, the forecast errors $x$ can be mapped to the uniformly distributed variable

$$
y=F_{e r}(x) \sim U[0,1]
$$

As shown in Fig. 5, the mapped data follows the uniform distribution quite well, except in the tail of the distribution for high forecast errors. The reason is the high density of points close to the identity line, as discussed previously. These points were removed if the forecast was above 0.3 , but a large number of such points still remain for lower forecast values.

Once the (almost) uniform variable $y$ is obtained, this can be mapped to a normally distributed variable using the inverse of the cdf $F_{\mathcal{N}}(z)$ for the standard normal distribution:

$$
z=F_{\mathcal{N}}^{-1}(y)
$$

Having mapped the errors to normally distributed variables, the covariance matrix between the errors at different times and different wind farms can be fit. However, notice that in order to obtain 5-minute data we need to use the errors with 5-min time resolution, and not 30-min time resolution as used to fit the distributions. The forecasts are for the average power production during the 30 minute period ending with the time stamp of the forecast. Thus a 5-min forecast can be made by assuming constant production during the $30 \mathrm{~min}$ period. This forecast is then filtered using the same lowpass filter that was used to filter the production data, before the 5-min forecast error is calculated. The 5-min errors are then mapped to normally distributed variables as described previously. Fig. 4 shows the covariance matrix of the forecast errors, both for the original data (after lowpass-filtering) and after mapping the errors to normally distributed variables. Notice that the correlation decreases somewhat after mapping the variables. In order to better preserve the correlation of the errors, the mapping was done using forecast values that had been additionally filtered using a lowpass filter with cutoff frequency 0.1 hours $^{-1}$.

To generate $\mathrm{n}$ scenarios from the fitted model, the method is as follows:

1) Start with a 5-min wind power forecast, obtained by filtering the rectangular 5-min forecast as described above.

2) Generate $n$ samples $z$ of the multivariate normal distribution, using the fitted correlation matrix.

3) Map the values to a multivariate uniform normal distribution using the cdf of the standard normal distribution: $y=F_{\mathcal{N}}(z)$.

4) Map the values to errors using the inverse of the fitted error distribution: $x=F_{e r}^{-1}(y)$.

5) Construct the scenarios by adding the errors to the original forecast.

\section{Fitting High Frequency Component}

As shown in Fig. 1 there are considerable intra-hour fluctuations in the wind power production. Since the forecast error is fit using lowpass-filtered data with a cutoff frequency of 1 hour $^{-1}$ these fluctuations are not captured in the scenarios. However, the high-frequency fluctuations can be added using filtered white noise. In Fig. 6 the top left graph shows the frequency spectrum (fft) of the 5-min production data for 4 separate days. Although the amplitude of the spectra vary 

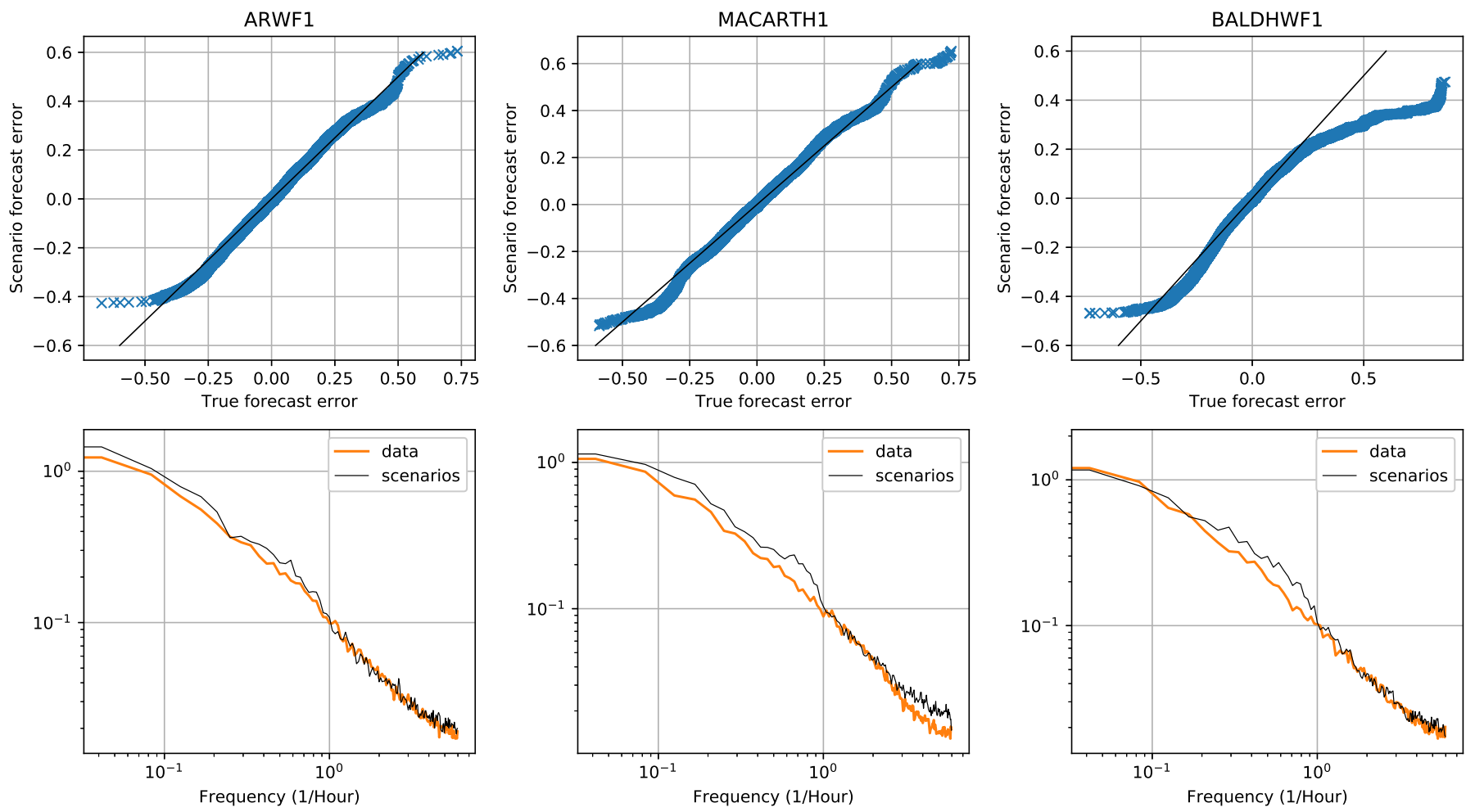

Figure 7. Out of sample validation for 100 scenarios generated from 100 forecasts (starting 2020-03-01) against the production data for the same days. The graphs on the top show the quantiles of the distribution of forecast errors obtained from the scenarios and the true forecast errors, and the graphs on the bottom show the average spectrum (fft) over the scenarios and the real production data.

significantly between the days, they are similar to a spectrum of white noise with an amplitude which varies as the inverse of the frequency.

Generally, the amplitude of the fluctuations are higher when wind production is higher. Thus, to fit noise of appropriate amplitude, the 24-hour periods were divided into three bins, after the average value of the forecasted wind production during period. Fig. 6 shows the results for the $3^{\text {rd }}$ bin, i.e. the $33 \%$ of days with the highest forecasted wind production. By taking all spectra in a bin and fitting a linear curve an "average" amplitude of the noise was obtained, as shown by the dashed line in the top right graph in Fig. 6. This could then be used to scale the white noise in the frequency domain. Then, the low-frequency part of the noise was removed by subtracting the lowpass filtered noise, using the same filter as in Section III. The spectrum of the resulting noise is shown in Fig. 6. This noise was then added to the original low-pass filtered signal, in order to get a new time series with the same low-frequency component but a different high-frequency component. The bottom part of Fig. 6 shows the original production data for a 24 hour period as well as four different high-frequency components.

\section{VAlidation AND ApPliCATION OF MODEL}

Fig. 7 shows an out of sample validation of the fitted model, using 100 days of data starting from 2020-03-01. For each day, one scenario was generated, giving a total of 100 scenarios which were compared to the actual wind power production on the same days. For both the scenarios and the actual production, the forecast errors were computed. The top row of Fig. 7 shows the quantiles of the generated forecast errors and the true forecast errors, where a curve close to the identity line indicates similar distributions. The plots show that the tails of the generated forecast errors are smaller (fewer values with very large forecast errors) than the true forecast errors, but this is partly by design as the fitted distributions were cut off at the $1 \%$ and $99 \%$ quantiles, and some outliers were omitted when fitting the distribution.

In the bottom row of Fig. 7 the average spectra of the scenario forecast errors and the true forecast errors are shown. When comparing the spectra of the generated and true forecast errors it was observed that the generated errors had higher spectral content below 1 hour $^{-1}$ than the actual data. This was attributed to the fact that the forecast error generated from the covariance matrix also had significant spectral content below this frequency, as it was generated with a 5-min time resolution. Thus the noise was reduced to $30 \%$ of its initial amplitude to get better agreement for the spectral content of the scenarios. It would also be possible to improve the noise modelling using techniques that better account for the variable high-frequency volatility of wind power production, e.g., the method proposed in [21].

The model can be used to generate an arbitrary number of 5-minute resolution, 24-hour duration scenarios from a given 


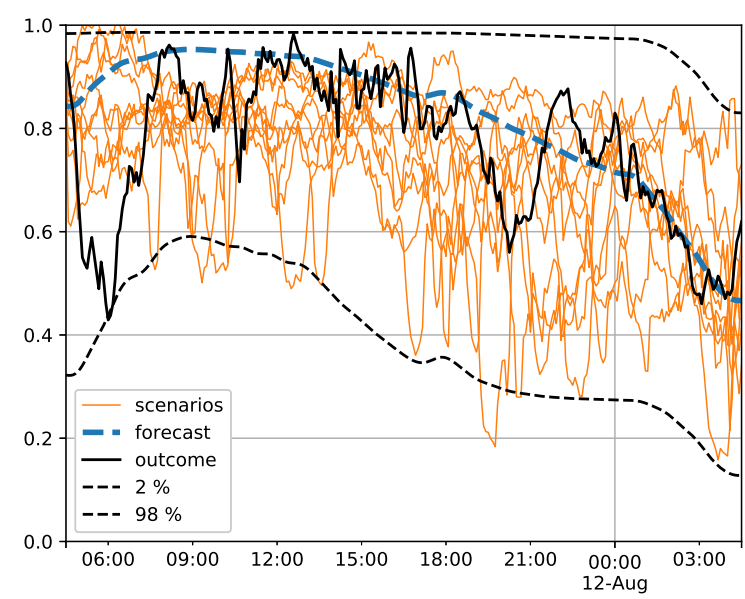

Figure 8. Forecasted wind production along with actual production and 10 scenarios for ARWF1. The dashed lines show the $2 \%$ and $98 \%$ quantiles for production, obtained from the parameterized error distribution.

historical forecast, as shown in Fig. 8. The scenarios may be used directly in the formulation of a stochastic UC/OPF formulation (hourly scenarios can be easily generated from the 5 -min scenarios by averaging), or used for doing out-of-sample evaluations of formulations. The model can also be used for obtaining the uncertainty range for use in robust formulations. Since the error distribution is known it is possible to obtain curves corresponding to certain quantiles for a given forecast, as shown in Fig. 8 for the 2\% and 98\% quantiles. Thus the robustness of a robust formulation can be set by choosing the quantiles to use for the uncertainty range, and the scenarios generated can be used for out-of-sample evaluation. The full model is available on Github ${ }^{1}$.

\section{CONCLUSION}

In the literature on unit commitment (UC) and optimal power flow $(\mathrm{OPF})$ with uncertainty there is significant variety relating to how the uncertainty from wind power is modelled, and methods range from using standard statistical distributions to more detailed modelling. In most cases the intra-hour variability of wind power generation is also not considered. In this paper we describe a method for generating realistic 5 -min resolution wind power scenarios, which consider the empirical forecast error distribution and correlation between different wind farms. We provide an open model and the data needed for users to generate an arbitrary number of wind power scenarios for use in stochastic optimization or outof-sample evaluation, or to generate uncertainty bounds for robust optimization methods. By providing this model we hope to facilitate comparison of different UC/OPF formulations, thereby contributing to their development.

\section{REFERENCES}

[1] P. A. Ruiz, C. R. Philbrick, E. Zak, K. W. Cheung, and P. W. Sauer, "Uncertainty management in the unit commitment problem," IEEE Trans. Power Syst., vol. 24, no. 2, pp. 642-651, 2009.
[2] Q. P. Zheng, J. Wang, and A. L. Liu, "Stochastic optimization for unit commitment-a review," IEEE Trans. Power Syst., vol. 30, no. 4, pp. 1913-1924, 2015.

[3] L. Roald, "Optimization methods to manage uncertainty and risk in power systems operation," Ph.D. dissertation, ETH Zurich, 2016.

[4] A. Papavasiliou, S. S. Oren, and R. P. O'Neill, "Reserve requirements for wind power integration: A scenario-based stochastic programming framework," IEEE Trans. Power Syst., vol. 26, no. 4, pp. 2197-2206, 2011.

[5] L. Wu, M. Shahidehpour, and Z. Li, "Comparison of scenario-based and interval optimization approaches to stochastic scuc," IEEE Trans. Power Syst., vol. 27, no. 2, pp. 913-921, 2012.

[6] C. Sahin, M. Shahidehpour, and I. Erkmen, "Allocation of hourly reserve versus demand response for security-constrained scheduling of stochastic wind energy," IEEE Trans. Sustain. Energy, vol. 4, no. 1, pp. 219-228, 2013.

[7] C. Uçkun, A. Botterud, and J. R. Birge, "An improved stochastic unit commitment formulation to accommodate wind uncertainty," IEEE Trans. Power Syst., vol. 31, no. 4, pp. 2507-2517, 2016.

[8] Q. Wang, Y. Guan, and J. Wang, "A chance-constrained two-stage stochastic program for unit commitment with uncertain wind power output," IEEE Trans. Power Syst., vol. 27, no. 1, pp. 206-215, 2012.

[9] Y. Wang, S. Zhao, Z. Zhou, A. Botterud, Y. Xu, and R. Chen, "Risk adjustable day-ahead unit commitment with wind power based on chance constrained goal programming," IEEE Trans. Sustain. Energy, vol. 8, no. 2, pp. 530-541, 2017.

[10] K. Sundar, H. Nagarajan, L. Roald, S. Misra, R. Bent, and D. Bienstock, "Chance-constrained unit commitment with $\mathrm{N}-1$ security and wind uncertainty," IEEE Trans. Control Netw. Syst., vol. 6, no. 3, pp. 10621074, Sep. 2019.

[11] C. Zhao, J. Wang, J. Watson, and Y. Guan, "Multi-stage robust unit commitment considering wind and demand response uncertainties," IEEE Trans. Power Syst., vol. 28, no. 3, pp. 2708-2717, 2013.

[12] Z. Zhang, Y. Chen, X. Liu, and W. Wang, "Two-stage robust securityconstrained unit commitment model considering time autocorrelation of wind/load prediction error and outage contingency probability of units," IEEE Access, vol. 7, pp. 25 398-25408, 2019.

[13] B. Hu and L. Wu, "Robust scuc considering continuous/discrete uncertainties and quick-start units: A two-stage robust optimization with mixed-integer recourse," IEEE Trans. Power Syst., vol. 31, no. 2, pp. 1407-1419, 2016.

[14] N. G. Cobos, J. M. Arroyo, and A. Street, "Least-cost reserve offer deliverability in day-ahead generation scheduling under wind uncertainty and generation and network outages," IEEE Trans. Smart Grid, vol. 9, no. 4, pp. 3430-3442, 2018.

[15] P. Xiong, P. Jirutitijaroen, and C. Singh, "A distributionally robust optimization model for unit commitment considering uncertain wind power generation," IEEE Trans. Power Syst., vol. 32, no. 1, pp. 39-49, 2017.

[16] G. Morales-España, Álvaro Lorca, and M. M. de Weerdt, "Robust unit commitment with dispatchable wind power," Electr. Power Syst. Res., vol. 155, pp. $58-66,2018$.

[17] G. Morales-España, R. Baldick, J. García-González, and A. Ramos, "Power-capacity and ramp-capability reserves for wind integration in power-based UC," IEEE Trans. Sustain. Energy, vol. 7, no. 2, pp. 614 624, 2016.

[18] “AEMO Market Data NEMWEB," https://www.aemo.com.au/energysystems/electricity/national-electricity-market-nem/data-nem/marketdata-nemweb, accessed on 28/11/2020.

[19] P. Pinson, H. Madsen, H. A. Nielsen, G. Papaefthymiou, and B. Klöckl, "From probabilistic forecasts to statistical scenarios of short-term wind power production," Wind Energy, vol. 12, no. 1, pp. 51-62, 2009.

[20] R. Koenker and K. F. Hallock, "Quantile regression," J. Econ. Perspect., vol. 15, no. 4, pp. 143-156, 2001.

[21] J. Olauson, H. Bergström, and M. Bergkvist, "Restoring the missing high-frequency fluctuations in a wind power model based on reanalysis data," Renew. Energy, vol. 96, pp. 784-791, 2016. 